Research Article

\title{
Bovine Tuberculosis in Grenada, West Indies: Current Status
}

\author{
Keshaw Tiwari ${ }^{1}$, Alfred Chikweto ${ }^{1}$, Derek Thomas ${ }^{3}$, Harry Hariharan ${ }^{1}$, \\ Raymond $\mathrm{Sis}^{2}$ and Ravindra Sharma ${ }^{1}$ \\ ${ }^{1}$ Pathobiology Academic Program, School of Veterinary Medicine, St. George's University, Grenada, \\ West Indies \\ ${ }^{2}$ School of Veterinary Medicine, St. George's University, Grenada, West Indies \\ ${ }^{3}$ Ministry of Agriculture, Fisheries and Forestry, Government of Grenada, West Indies
}

Correspondence should be addressed to: Harry Hariharan; hhariharan@sgu.edu

Received 21 May 2013; Accepted 19 June 2013; Published 28 Septemeber 2013

Academic Editor: Viviana Ritacco

Copyright (C) 2013 Keshaw Tiwari, Alfred Chikweto, Derek Thomas, Harry Hariharan, Raymond Sis and Ravindra Sharma. Distributed under Creative Commons CC-BY 3.0

\begin{abstract}
A study was conducted to determine the prevalence of bovine tuberculosis in Grenada, West Indies, using an intradermal tuberculin test and a commercial bovine interferon gamma (IFN- $\gamma$ ) ELISA simultaneously. All 148 randomly selected cattle comprising 10\% of the estimated cattle population of Grenada, gave negative results in both tests, indicating possible freedom from tuberculosis in cattle herds in this island nation.
\end{abstract}

Keywords: Tuberculosis, bovine, Grenada.

\section{Introduction}

Bovine tuberculosis caused by Mycobacterium bovis is found in cattle throughout the globe. A broad range of mammalian hosts; including humans, cattle, deer, llamas, cats, pigs, wild carnivores (fox, coyotes) and omnivores (opossums, rodents, and mustlids), and rarely equines and sheep can be infected by M. bovis (Delahay et al., 2002).

The incidence of disease in bovines varies from one country to another depending on several factors, including location of the country, wildlife reservoirs, and national control programs of herd tuberculin testing, supported by active abattoir surveillance. Through the process of "test and cull" many countries in the world have been able to reduce or limit its incidence. Status of bovine tuberculosis in the Caribbean countries has been reported by de Kantor and Ritacco (1994). According to them 12 countries in Caribbean, namely Anguilla, Bahamas, Barbados, Bermudas, British Virgin Islands, Guadeloupe, Montserrat, Saint Kits and Nevis, Saint Lucia, Saint Vincent, Saint Pierre, and Trinidad-Tobago are free of bovine tuberculosis. Cuba was declared free from bovine tuberculosis in 1984; in Jamaica cattle carcass condemnation due to tuberculosis was less than $0.01 \%$, whereas

Cite this Article as: Keshaw Tiwari, Alfred Chikweto, Derek Thomas, Harry Hariharan, Raymond Sis and Ravindra Sharma (2013), "Bovine Tuberculosis in Grenada, West Indies: Current Status," International Journal of Veterinary Medicine: Research \& Reports, Vol. 2013 (2013), Article ID 169877, DOI: $10.5171 / 2013.69877$ 
in Haiti, there were no carcass condemnations due to tuberculosis over a period of five years in the late 1980s. In Dominican Republic the eradication program for tuberculosis and brucellosis started in 1974 and by 2001 prevalence of tuberculin reactors was as low as $0.63 \%$ (de Kantor and Ritacco, 2006). Several Caribbean islands had not reported bovine TB (Ritacco et al., 2008). In Grenada, intradermal tuberculin test conducted on cattle by the United States army in 1996 did not show any positive reactors (Louison, B., Chief Veterinary Officer, Grenada, personal communication, 2013).

Tuberculin testing has been in use for several decades for control of bovine tuberculosis. Recently, a commercial test, bovine interferon gamma (IFN- $\gamma$ ) assay has been approved for use within the United States as a complementary test for tuberculosis. This assay has also been approved by the Office International des Epizooties (OIE/World organization for Animal Health) as an ancillary test to confirm or negate the results of intra dermal tuberculin test (Prionics, 2008).

The objective of the present study was to estimate the prevalence of tuberculosis in cattle herds in Grenada using both intra dermal tuberculin test and IFN- $\gamma$ assay.

\section{Methods}

Blood from jugular vein was collected in two separate tubes with heparin from 148 randomly sampled cattle from all the six parishes of mainland Grenada (35 herds, consisting of $6 \%$ of the estimated cattle population in the country, which is approximately 2500). All cattle were sampled from herds having less than 10 cattle. When the herd size was more than 10 cattle, $80 \%$ of animals were sampled. In general, most herds in Grenada are small, consisting of 1-3 animals.

To perform the IFN- $\gamma$ assay, avian and bovine tuberculin (Prionics AG, Schlieren, and Switzerland) were added in whole blood samples (in separate tubes) within 5 minutes of blood collection, and incubated at $37^{\circ} \mathrm{C}$ overnight. After incubation, plasma was harvested by centrifugation for 5 minutes at $2000 \mathrm{~g}$. Plasma was tested with IFN- $\gamma$ ELISA (Bovigam ELISA kit from Prionics AG, Switzerland) as per the manufacturer's directions. Concurrently, an intradermal tuberculin test, using tuberculin PPD (bovine) and tuberculin PPD (avian), obtained from Prionics USA (La Vista, Nebraska), was performed on each corresponding animal from which blood was collected for IFN- $\gamma$ ELISA. For tuberculin test, injection sites selected for PPD (bovine) and PPD (avian) were in the middle third of the side of the neck, one above the other separated by about 10-12 $\mathrm{cm}$. The animals were ear tagged for the purpose of identification.

An interviewer-administered questionnaire was used to get information about awareness of bovine tuberculosis by cattle owners. A total of 33 farmers were interviewed.

\section{Results and Discussion}

Of the 148 cattle tested, no animal gave a positive reaction for bovine tuberculosis in intradermal tuberculin test or in IFN- $\gamma$ ELISA. It is accepted that IFN- $\gamma$ ELISA detects animals that show a negative reaction with intradermal tuberculin test, probably because it detects animals earlier after infection than tuberculin test. When used in parallel with tuberculin test, interferon gamma assay is capable of identifying infected cattle, which might otherwise not be detected until later (Gormley et al., 2006). The IFN- $\gamma$ assay is at least as sensitive and specific as the best considered intradermal tuberculin tests, and it performs with high accuracy in the field (de Kantor and Ritacco, 2006, Antognoli et al., 2011). However, its high cost hampers its use in routine diagnostic screening for tuberculosis (Ritacco et al., 1991; Linenbaum et al., 1999; Perez et al., 2002). The advantage of early detection by using a combination of the above tests is that positive animals can be removed, resulting in reduction of the source of infection for other cattle. Tuberculin test in cattle does not interfere with IFN- $\gamma$ response. However, false-negative response for tuberculin test can occur soon 
after infection, in the late stage of the disease, and in animals with poor immune response (Tizard, 2013).

Of the 33 cattle farmers interviewed, 6 (18\%) had heard about bovine tuberculosis. Out of these 6 , four $(66.7 \%)$ knew how it was transmitted and only one $(16.7 \%)$ knew of its zoonotic potential. Twenty seven cattle owners were not aware of this important disease. None of the farmers reported their animal carcass condemned at the abattoir as a result of lesions consistent with bovine tuberculosis. It has been reported that the level of disease awareness among farmers is often related to the prevalence of the disease (Brook and McLachlan, 2006). Therefore, the low level of awareness among cattle owners on bovine tuberculosis in Grenada may be related to the apparent freedom from this disease in the country. It may be noted that the cattle herds in Grenada are small, and there is no wildlife such as deer, elk, and bison, which could serve as reservoirs of tuberculosis in this island nation. Published information on human tuberculosis in Grenada is lacking, although cases of tuberculosis have been reported from the prison in Grenada (Thomas D, Ministry of Agriculture, Fisheries and Forestry, Government of Grenada, personal communication. 2013). In Trinidad and Tobago, 17 cases of human tuberculosis were reported in the year 2006 from a population of 100,000 (Baboolal et al., (2009). However, human tuberculosis caused by $M$. bovis appears to be rare in most regions of the Americas, including the Caribbean (de Kantor et al., (2010). In the United States, human tuberculosis due to M. bovis during 1995 to 2005 was only $1.4 \%$ among cases confirmed by bacteriological tests (Hlavsa et al., 2008). Similarly, in the United Kingdom, only 0.5 to $1.5 \%$ of all culture-confirmed cases were due to M. bovis during 1990 to 2003 (dela Rua-Domenech, 2006). In Latin America, less than $1 \%$ of human tuberculosis is reported as being due to $M$. bovis (Thoen et al., 2009). Further studies, including laboratory examination of lymph nodes from carcasses of slaughtered animals may shed more light on the prevalence of this important zoonotic disease in Grenada.

\section{Conclusions}

A study conducted two decades ago in Grenada for bovine tuberculosis using the intradermal tuberculin test gave negative results indicating absence of this disease in cattle. Our results of this study using the intradermal tuberculin test and an additional test, an IFN- $\gamma$ ELISA, confirm that bovine tuberculosis does not appear to be a problem at present in Grenada. However, continued surveillance, including examination of suspected specimens from slaughtered cattle is required to establish freedom from this important zoonotic disease.

\section{References}

Antognoli, M. C., Remmenga, M. D., Bengtson, S. D., Clark, H. J., Orloski, K. A., Gustafson, L. L. \& Scott, A. E. (2011). "Analysis of the Diagnostic Accuracy of the Gamma Interferon Assay for Detection of Bovine Tuberculosis in U.S. Herds," Preventive Veterinary Medicine 101 (1-2) 35-41.

Baboolal, S., Millet, J., Akpaka, P. E., Ramoutar, D. \& Rastogi, N. (2009). "First Insight into Mycobacterium Tuberculosis Epidemiology and Genetic Diversity in Trinidad and Tobago," Journal of Clinical Microbiology 47: 1911-1914.

Brook, R. K. \& Mclachlan, S. M. (2006). "Factors Influencing Farmer's Concerns Regarding Bovine Tuberculosis in Wildlife and Livestock around Riding Mountain National Park," Journal of Environmental Management 80 (2) 156-166.

De Kantor, I. N., La Bue, P. A. \& Thoen, C. O. (2010). "Human Tuberculosis Caused by Mycobacterium Bovis in the United States, Latin America, and the Caribbean," International Journal of Tuberculosis and Lung Diseases. 14: 1369-1373.

De Kantor, I. N. \& Ritacco, V. (1994). "Bovine Tuberculosis in Latin America and the Caribbean: Current Status, Control and Eradication Programs," Veterinary Microbiology 40 (1-2) 5-14. 
De Kantor, I. N. \& Ritacco, V. (2006). "An Update on Bovine Tuberculosis Programs in Latin America and Caribbean Countries," Veterinary Microbiology 112 (2-4) 111-118.

De La Rua-Domenech, R. (2006). "Human Mycobacterium Bovis Infection in the United Kingdom: Incidence, Risks, Control Measures and Review of the Zoonotic Aspects of Bovine Tuberculosis," Tuberculosis (Edinburgh) 86: 77-109.

Delahay, R. J., De Leeuw, A. N. S., Barlow, A. M., Cliffon-Hadley, R. S. \& Cheeseman, C. L. (2002). "The Status of Mycobacterium Bovis Infection in UK Wild Mammals: A Review," The Veterinary Journal 164 (2) 90105.

Gormley, E., Doyle. M. B., Fitzsimons, T., Mcgill, K. \& Collins, J. D. (2006). "Diagnosis of Mycobacterium Bovis Infection in Cattle by Use of the Gamma-Interferon (Bovigam) Assay," Veterinary Microbiology, 112 (2-4) 171-179.

Halvsa, M. C., Moonan, P. K., Cowan, L. S., Navin, T. R., Kammerer, J. S., Morlock, G. P., Crawford, J. T. \& Lobue, P. A. (2008). "Human Tuberculosis Due to Mycobacterium Bovis in the United States, 1995-2005," Clinical Infectious Diseases 47:168-175.

Lilenbaum, W., Schettini,J. C., Souza, G. N., Ribeiro, E. R., Moreira, E. C. \& Fonseca, L. S. (1999). "Comparison between a GammaIFN Assay and Intradermal Tuberculin Test for the Diagnosis of Bovine Tuberculosis in Field Trials in Brazil," Zentralblatt Fur Veterinarmedizin Reihe B 46 (5) 353-358.

Perez, A. M.,Ward, M. P., Charmandarian, A. \& Ritacco,V. (2002). "Simulation Model of Within-Herd Transmission of Bovine Tuberculosis in Argentine Dairy Herds," Preventive Veterinary Medicine, 54 (4) 361372.

Prionics. (2008).

http://www.prionics.com/index.php?id=8 4

Ritacco, V., Lopez, B., Dekantor, I. N., Barrera, L., Errico, F. \& Nader, A. (1991).
"Reciprocal Cellular and Humoral Immune Responses in Bovine Tuberculosis," Research in Veterinary Science. 50 (3) 365367.

Ritacco V., Torres, P., Sequeira, M. D., Reniero, A. \& De Kantor, I. N. (2008). Bovine Tuberculosis in Latin America and Caribbean. In: Mycobacterium Bovis: Infections in Animals and Humans. 2nd Ed. Wiley Publishers, Hoboken, N.J., USA. 149160.

Thoen, C. O., Lobue, P. A., Enarson, D. A., Kaneene, J. B. \& De Kantor, N. (2009). "Tuberculosis: A Re-Emerging Disease in Animals and Humans," Veterinaria Italiana. 45: 135-181.

Tizard, I. R. (2013). 'Veterinary Immunology,' 9th Ed. Elsevier/Saunders, St. Louis, Missouri. 637. 